Quim. Nova, Vol. 34, No. 3, 408-413, 2011

\title{
PREPARAÇÃO E CARACTERIZAÇÃO DE SUSPENSÕES COLOIDAIS DE NANOCARREADORES LIPÍDICOS CONTENDO RESVERATROL DESTINADOS À ADMINISTRAÇÃO CUTÂNEA
}

\author{
Daiane Nemen e Elenara Lemos-Senna* \\ Departamento de Ciências Farmacêuticas, Centro de Ciências da Saúde, Universidade Federal de Santa Catarina, Campus Trindade, \\ CP 476, 88040-900 Florianopolis - SC, Brasil
}

Recebido em 19/5/10; aceito em 20/10/10; publicado na web em 31/1/11

\begin{abstract}
PREPARATION AND CHARACTERIZATION OF RESVERATROL-LOADED LIPID-BASED NANOCARRIERS FOR CUTANEOUS ADMINISTRATION. Solid lipid nanoparticles (SLN), nanoemulsions (NE), and microemulsions (ME) were prepared by the hot solvent diffusion method, using tristearin or castor oil as oily phase, and soy lecithin and Solutol HS 15 as surfactants. Mean particle sizes ranged from 20 to $215 \mathrm{~nm}$ and negative zeta potentials were obtained for all nanocarriers. A HPLC method used to determine resveratrol was specific, linear, exact and precise. The entrapment efficiency was high for all formulations. However, resveratrol content was significantly varied among the lipid nanocarriers. Lipid nanocarrier containing hydrogels exhibiting pseudoplastic behavior were obtained after incorporation of hydroxyethylcellulose in the colloidal dispersions.
\end{abstract}

Keywords: resveratrol; lipid-based nanocarriers; hydrogels.

\section{INTRODUÇÃO}

O resveratrol (3,4,5-tri-hidroxiestilbeno) (Figura 1) é uma fitoalexina presente numa grande variedade de espécies vegetais, incluindo amoras, amendoins e uvas, e, portanto, é um constituinte da dieta humana. Este composto, tal como outros membros da família do estilbeno, é produzido nos vegetais em resposta ao ataque de patógenos, à radiação ultravioleta e à exposição ao ozônio. A Vitis vinifera, ou a uva, sintetiza o resveratrol como meio de proteção às infecções fúngicas, sendo, portanto, encontrado em altas concentrações no vinho. ${ }^{1,2}$

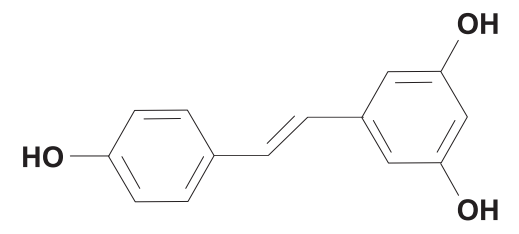

Figura 1. Estrutura química do trans-resveratrol

Vários estudos têm destacado e comprovado os benefícios do resveratrol à saúde. Esta substância tem demonstrado propriedades quimiopreventivas, ${ }^{3-8}$ antioxidante, ${ }^{9,10}$ antiplaquetária, ${ }^{11}$ antifúngica, ${ }^{12}$ anti-inflamatória, ${ }^{13}$ cardioprotetora, ${ }^{14,15}$ entre outras. $\mathrm{O}$ resveratrol age como efetor biológico pleiotrópico que regula as três fases do processo da carcinogênese, ou seja, início, promoção e progressão do tumor. ${ }^{1}$ Uma vez absorvidos, os agentes quimiopreventivos podem agir por diferentes mecanismos, seja pela inibição da ativação metabólica de compostos carcinogênicos, estímulo da detoxificação de metabólitos reativos, prevenção da sua interação com o DNA da célula e supressão da progressão do tumor. Experimentos in vitro, ex vivo e in vivo têm demonstrado que além de atuar nos mecanismos quimiopreventivos descritos acima, o resveratrol também possui potencial quimioterapêutico. De fato, o resveratrol suprime o crescimento de várias linhagens de células cancerosas, em parte

*e-mail: lemos@ccs.ufsc.br pela inibição da DNA polimerase e ribonucleotídeo redutase ou pela indução da suspensão do ciclo celular ou apoptose. ${ }^{16}$ Os efeitos anticarcinogênicos provocados pelo resveratrol também parecem estar associados à sua atividade antioxidante, visto que ele tem demonstrado inibir a ciclo-oxigenase, hidroperoxidase, proteína quinase $\mathrm{C}$, fosforilação da Bcl-2, proteína quinase B (PKB ou Akt), quinase de adesão focal, NF- $\kappa B$, metaloprotease de matriz tipo 9, e reguladores do ciclo celular. ${ }^{17} \mathrm{O}$ resveratrol também inibe a oxidação da LDL, por modular a atividade do metabolismo de lipídeos e lipoproteínas, 2,18 suprime a angiogênese ${ }^{18}$ e apresenta atividade estrogênica devido a sua semelhança estrutural com o agente estrogênico dietilestilbestrol. ${ }^{2}$

Apesar dos numerosos estudos sobre as propriedades dos compostos polifenólicos in vitro, estes efeitos frequentemente não são observados in vivo. A aparente discrepância entre estudos in vitro e in vivo pode ser parcialmente atribuída à baixa absorção e elevado metabolismo destes compostos. ${ }^{19}$ Após administração oral na dose de $25 \mathrm{mg}$, a absorção do resveratrol demonstrou ser de pelo menos $70 \%$. Entretanto, a sua biodisponibilidade foi quase nula; unicamente traços da molécula na forma inalterada foram encontrados no plasma. A rápida conjugação com sulfato no intestino e fígado foi responsável pela baixa biodisponibilidade oral do resveratrol. ${ }^{20,21}$ Outros fatores que limitam o aproveitamento terapêutico do resveratrol incluem a sua baixa estabilidade frente à oxidação e baixa solubilidade aquosa. ${ }^{22}$

Inúmeros estudos relatam os benefícios do resveratrol sobre a pele, fazendo com que a liberação tópica deste composto constitua uma interessante alternativa à administração oral para o tratamento de várias desordens cutâneas. Estudos evidenciaram fortes propriedades antiproliferativas e quimiopreventivas contra a carcinogênese, ${ }^{23-26}$ proteção contra os danos causados pela radiação ultravioleta, e atividade antimicrobiana contra dermatófitos e ao vírus da Herpes simplex. ${ }^{27,28}$ Apesar dos estudos de permeabilidade cutânea terem sido promissores, nenhuma evidência tem sido dada quanto à obtenção de concentrações terapêuticas deste fármaco na pele, sugerindo que outros sistemas de liberação possam ser testados com o intuito de potencializar a permeação cutânea e obter preparações eficazes para o tratamento de desordens cutâneas. ${ }^{29}$ Portanto, o desenvolvimento 
de sistemas nanocarreadores pode ser considerado uma interessante abordagem para explorar as propriedades deste composto na clínica.

Carreadores coloidais diferem entre si em função da sua composição, capacidade de incorporar fármacos e aplicabilidade, mas a característica comum é o tamanho de partícula submicrométrico. Entre os carreadores nanoestruturados com potencial aplicação tópica destacam-se as nanopartículas lipídicas sólidas (NLS), as nanoemulsões (NE) e as microemulsões (ME). Estes sistemas nanoestruturados são constituídos por lipídios altamente purificados e misturas complexas de glicerídeos apresentando tamanho de partícula variável. Suas principais características incluem excelente estabilidade física, capacidade de proteção de fármacos instáveis frente à degradação, capacidade de controle da liberação, excelente tolerabilidade, possibilidade de vetorização, além de não apresentar problemas relacionados à produção em grande escala e à esterilização. ${ }^{30-32}$ Enquanto as NLSs são sistemas matriciais organizados obtidos a partir de triglicerídeos sólidos, as NEs submicrônicas são obtidas como dispersões óleo em água, apresentando elevada estabilidade cinética, em decorrência do seu reduzido tamanho de gota. As MEs, por outro lado, são sistemas isotrópicos, transparentes, de baixa viscosidade e termodinamicamente estáveis, obtidas quando uma mistura de surfactantes apropriada é usada ${ }^{33,34}$ Neste trabalho, a capacidade destes sistemas em incorporar o resveratrol foi avaliada, visando o seu aproveitamento terapêutico no tratamento de patologias cutâneas. Para tal, a hidroxietilcelulose foi utilizada para conferir viscosidade adequada às formulações. Existem muitos relatos descrevendo métodos para quantificação do resveratrol. Entretanto, a maioria deles tem sido focada em determinações simultâneas de vários compostos do vinho ${ }^{2,35,36}$ e identificação de metabólitos do resveratrol em plasma humano e animal. ${ }^{20,37,38}$ Assim, este trabalho também descreve o desenvolvimento e validação de um método de cromatografia líquida de alta eficiência para a determinação do resveratrol nas suspensões coloidais e nos hidrogeis obtidos após a incorporação da hidroxietilcelulose.

\section{PARTE EXPERIMENTAL}

\section{Materiais}

O trans-resveratrol (3,5,4'-tri-hidroxi-trans-estilbeno, aproximadamente $99 \%$ de pureza) foi adquirido da Sigma (St. Louis, USA). O óleo de rícino e a lecitina de soja hidrogenada (Lecitina S75-3N ${ }^{\circledR}$ ) foram adquiridos da Via Pharma (São Paulo, Brasil) e SP Pharma (São Paulo, Brasil), respectivamente. Hidroxietilcelulose (HEC) (Natrosol $^{\circledR}$ ) foi adquirida da Denver-Cotia (São Paulo, Brasil). A triestearina (Dynasan 118) foi gentilmente doada pela Sasol (Louisiana, EUA) e o hidroxiestearato de polietilenoglicol (Solutol HS-15 ${ }^{\circledR}$ ) foi doado pela Basf (Trostberg, Alemanha). Água ultrapura foi fornecida por sistema de purificação Milli-Q ${ }^{\circledR}$ (Millipore, Bedford, EUA). Metanol grau HPLC foi adquirido da J.T. Baker ${ }^{\circledR}$ (Phillipsburg, EUA). Etanol, acetona e outros reagentes químicos usados foram de grau analítico.

\section{Preparação das suspensões coloidais de nanocarreadores lipídicos}

Os nanocarreadores lipídicos (NLS, NE e ME) foram preparados pelo método de difusão do solvente a quente..$^{39}$ Resumidamente, 10 mg de lecitina e $100 \mathrm{mg}$ do lipídio (triestearina ou óleo de rícino) foram dissolvidos em uma mistura de acetona:etanol $(60: 40, \mathrm{v} / \mathrm{v})$ a $60{ }^{\circ} \mathrm{C}$. A solução orgânica resultante foi rapidamente adicionada em 50 mL de uma solução aquosa contendo Solutol HS $15^{\circledR}(0,1 \%$ para a obtenção das NLS e NE, ou 1,0\% para a obtenção da ME), mantida sob agitação magnética a $82^{\circ} \mathrm{C}$. A suspensão coloidal obtida foi então resfriada até temperatura ambiente, o solvente orgânico evaporado sob pressão reduzida e o volume final foi ajustado para $20 \mathrm{~mL}$. Finalmente, a suspensão coloidal foi filtrada através de filtro de papel quantitativo de $8 \mu \mathrm{m}$ (J. Prolab, Brasil).

Para a preparação dos nanocarreadores lipídicos contendo resveratrol $\left(\mathrm{NLS}_{\mathrm{rv}}, \mathrm{NE}_{\mathrm{rv}}\right.$ e $\left.\mathrm{ME}_{\mathrm{rv}}\right)$, o fármaco foi adicionado na fase orgânica das formulações em diferentes concentrações. As formulações foram preparadas em triplicata. O efeito do tipo de nanocarreador lipídico e da quantidade inicial de resveratrol sobre o teor de fármaco nas suspensões coloidais foi avaliado por meio da análise da variança de duas vias usando o programa StatPlus ${ }^{\circledR}$ Profissional (AnalystSoft, Inc.).

\section{Preparação dos hidrogeis}

Para a obtenção de geis contendo os diferentes nanocarreadores lipídicos, $300 \mathrm{mg}$ de hidroxietilcelulose $\left(\mathrm{Natrosol}^{\circledR}\right)$ foram adicionados a $20 \mathrm{~mL}$ de cada uma das suspensões coloidais contendo resveratrol. As misturas foram mantidas sob agitação magnética durante $12 \mathrm{~h}$ em temperatura ambiente e posteriormente armazenadas até análise. Hidrogeis contendo sistemas nanoestruturados sem fármaco foram preparados da mesma maneira.

\section{Caracterização físico-química das suspensões coloidais}

O tamanho médio e o potencial zeta das suspensões coloidais foram determinados por espectroscopia de correlação fotônica e anemometria laser Doppler, respectivamente, utilizando um Zetasizer Nano Series (Malvern Instruments, Worcestershire, UK). As medidas foram realizadas a $25^{\circ} \mathrm{C}$, após diluição apropriada das amostras em água destilada. Cada análise de tamanho durou $120 \mathrm{~s}$ e foi obtida com um ângulo de detecção de $173^{\circ}$. Para determinar o potencial zeta, as amostras foram colocadas em célula eletroforética, onde um potencial de $\pm 150 \mathrm{mV}$ foi estabelecido. Os valores potenciais foram calculados como a média dos valores da mobilidade eletroforética utilizando a Equação de Smoluchowski. ${ }^{40}$

\section{Determinação do resveratrol nas suspensões coloidais e hidrogeis por cromatografia líquida de alta eficiência (CLAE)}

\section{Condições instrumentais e analíticas}

A análise por cromatografia líquida de alta eficiência foi realizada em um equipamento Perkin Elmer S200 (Shelton, EUA) equipado com desgaseificador Série 200, autoamostrador Série 200, detector UV-visível Série 200, bomba binária Série 200 e uma interface cromatográfica Série 600 Link. O comprimento de onda do detector foi fixado em $312 \mathrm{~nm}$ e a área do pico foi analisada automaticamente usando-se um programa de software Totalchrom. Os experimentos foram conduzidos em fase reversa usando uma coluna $\mathrm{C}_{18}$ Zorbax ODS (150 x 4,6 mm, $5 \mu \mathrm{m}$; Agilent Technologies, EUA), mantida a $24 \pm$ $1{ }^{\circ} \mathrm{C}$. A fase móvel foi constituída de uma mistura de metanol:água (47:53, v/v) e foi eluída em modo isocrático em um fluxo de $1 \mathrm{~mL} / \mathrm{min}$.

\section{Validação do método analítico}

Os parâmetros empregados para a validação do método de CLAE foram: especificidade, linearidade, exatidão, precisão, limites de quantificação e detecção. A validação foi realizada com base nas diretrizes da International Conference on Harmonization $(\mathrm{ICH})^{41} \mathrm{e}$ Farmacopeia Americana. ${ }^{42}$

\section{Especificidade}

A especificidade do método cromatográfico foi avaliada através da análise das suspensões coloidais e hidrogeis contendo nanocarreadores sem fármaco, a fim de verificar a possível interferência dos 
excipientes da formulação na quantificação do fármaco. A análise de cada uma das amostras foi realizada em triplicata.

\section{Linearidade}

Soluções padrão de resveratrol em metanol:água $(47: 53, \mathrm{v} / \mathrm{v})$ foram obtidas nas concentrações de 0,$4 ; 0,6 ; 0,8 ; 1 ; 2 ; 4 ; 6 ; 8$ e 10 $\mu \mathrm{g} / \mathrm{mL}$. As soluções foram injetadas no cromatógrafo em 3 replicatas cada e em 3 dias diferentes. Curvas de concentração de resveratrol em função da área do pico foram construídas e a linearidade do método na faixa de concentração testada foi avaliada pela análise da regressão linear.

\section{Limites de detecção e quantificação}

Os limites de detecção (LD) e quantificação (LQ) foram calculados diretamente a partir da inclinação (S) e do desvio padrão do intercepto (DP), obtidos após a construção de três curvas de calibração. O limite de detecção foi expresso como $L D=(3,3 \times D P) / S$ e o limite de quantificação como $L Q=(10 \times D P) / S$.

\section{Exatidão}

A exatidão foi investigada contaminando as suspensões coloidais brancas com concentrações conhecidas de resveratrol em três diferentes níveis (baixo, médio e alto), correspondendo às concentrações finais de 1,0;2,0 e 4,0 $\mu \mathrm{g} / \mathrm{mL}$. As amostras foram diluídas em metanol e centrifugadas a $5000 \mathrm{rpm}$. Uma alíquota do sobrenadante foi então coletada e diluída em uma mistura de metanol:água (47:53, v/v). As análises foram realizadas em triplicata. A exatidão foi expressa como porcentagem de resveratrol recuperado $(\mathrm{R} \%)$.

\section{Precisão}

A precisão do método foi determinada por medidas da repetibilidade (precisão intradia) e precisão intermediária (precisão interdia). A repetibilidade foi avaliada pela determinação de 6 diferentes amostras de suspensões dos nanocarreadores lipídicos contendo $4 \mu \mathrm{g} / \mathrm{mL}$ de resveratrol, no mesmo dia, sob as mesmas condições experimentais. A precisão intermediária foi avaliada através da realização da análise em 3 dias diferentes.

Para análise por CLAE, as amostras foram diluídas em metanol e centrifugadas a $5000 \mathrm{rpm}$. Uma alíquota do sobrenadante foi então coletada e diluída em uma mistura de metanol:água (47:53, v/v). As áreas dos picos foram determinadas e comparadas. A precisão foi expressa como percentagem do desvio padrão relativo (DPR\%).

\section{Determinação do teor e eficiência de encapsulação do resveratrol}

Para a determinação do teor de resveratrol (concentração total), uma alíquota de cada suspensão coloidal ou do hidrogel foi quantitativamente transferida para um balão volumétrico de $10,0 \mathrm{~mL}$ e o volume final foi completado com metanol. Após, as amostras foram centrifugadas e uma alíquota do sobrenadante foi diluída em uma mistura de metanol:água (47:53, v/v). As amostras foram injetadas no cromatógrafo em triplicata e as áreas médias dos picos referentes ao resveratrol foram comparadas com a área do pico de uma solução padrão de concentração conhecida. A concentração foi expressa em $\mu \mathrm{g}$ de resveratrol por $\mathrm{mL}$ de suspensão. A eficiência de encapsulação (EE $\%$ ) foi estimada como sendo a diferença entre a concentração total de resveratrol e aquela encontrada no ultrafiltrado, obtido após ultrafiltração/centrifugação das suspensões coloidais utilizando unidades de filtração Ultrafree - MC (100.000 MWCO, Millipore, EUA). A recuperação do resveratrol nas suspensões nanocarreadoras foi calculada como sendo o percentual da concentração total de fármaco encontrado nas suspensões em relação à quantidade inicial adicionada.

\section{Avaliação da viscosidade dos hidrogeis contendo nanocarreadores lipídicos}

A caracterização reológica dos hidrogeis foi realizada usando-se um viscosímetro rotacional Brookfield DV-II +, com adaptador de pequenas amostras e spindle SC4-18 (Brookfield Engineering Laboratories, Middleboro, EUA), acoplado ao software Rheocalc. As amostras de hidrogeis de resveratrol foram mantidas à temperatura ambiente e submetidas a velocidades de rotação de 5, 10, 20 e 30 rpm, respeitando os limites de torque (entre 10 e 100\%). Os valores de tensão de cisalhamento e viscosidade, em função do gradiente de cisalhamento, foram avaliados e comparados.

\section{Avaliação da morfologia}

Uma alíquota de cada um dos hidrogeis contendo nanocarreadores lipídicos brancos (sem fármaco) foi transferida para recipientes de teflon e colocados em estufa à $45^{\circ} \mathrm{C}$ até completa evaporação da água. Os filmes obtidos a partir dos hidrogeis foram fixados em porta-amostras e recobertos com uma fina camada de ouro. As amostras foram analisadas por microscopia eletrônica de varredura com canhão de emissão de campo (SEM-FEG) (Jeol JSM-6701F, Japão), com tensão de aceleração de 5,0 kVa.

\section{RESULTADOS E DISCUSSÃO}

A administração cutânea ainda é considerada um desafio na área farmacêutica, visto que o estrato córneo impõe uma barreira à passagem de fármacos, dificultando o alcance do mesmo nas diferentes camadas da pele. Diferentes estratégias têm sido testadas com o objetivo de romper a barreira imposta pela camada córnea e aumentar a passagem de fármacos através da pele, incluindo o emprego de sistemas nanoestruturados lipídicos. ${ }^{43,44} \mathrm{O}$ aumento da superfície de contato do fármaco com os corneócitos, o efeito oclusivo provocado sobre a pele, a rápida e constante liberação do fármaco e a ação do surfactante sobre a permeabilidade parecem estar envolvidos no aumento da permeação cutânea de fármacos a partir destes nanocarreadores..$^{45,46}$ Assim, buscando obter formulações destinadas à administração tópica cutânea, a encapsulação do resveratrol em sistemas nanoestruturados lipídicos foi realizada usando o método de difusão do solvente a quente para a preparação das suspensões coloidais.

Neste trabalho foram empregados a triestearina e o óleo de rícino como lipídio sólido e líquido, para obtenção da fase oleosa da preparação, e a lecitina de soja e o Solutol HS 15, como surfactantes lipofílico e hidrofílico, respectivamente. Estudos anteriores realizados em nosso laboratório mostraram que em função da composição das formulações, ou seja, tipo de lipídio e concentração de Solutol HS 15 na fase aquosa, nanopartículas lipídicas sólidas (NLS), nanoemulsões (NE) e microemulsões (ME) podem ser obtidas. Neste caso, NLS e NE foram obtidas usando Solutol HS 15 0,1\%. As ME foram obtidas com o uso de Solutol HS $151 \% .^{47}$

\section{Validação da metodologia analítica}

A capacidade dos sistemas nanoestruturados lipídicos em incorporar o resveratrol foi avaliada e comparada, usando-se para isso os parâmetros de teor de fármaco e eficiência de encapsulação. Para tal, um método analítico de cromatografia líquida de alta eficiência foi desenvolvido e validado, de modo a garantir que ele gere informações confiáveis e interpretáveis das amostras. Diferentes proporções de metanol e água foram testadas como eluente, variando de 40:60 a 60:40 (v/v). O melhor desempenho cromatográfico foi alcançado quando a mistura de metanol:água 47:53 (v/v) foi empregada como 
eluente, com um fluxo de $1 \mathrm{~mL} / \mathrm{min}$. Nestas condições, o tempo de retenção do resveratrol foi em torno de 4,6 min. A especificidade do método foi avaliada por meio da análise das suspensões coloidais e dos hidrogeis contendo os nanocarreadores lipídicos sem fármaco. Os cromatogramas mostraram que o método é específico e não há nenhuma interferência ou sobreposição dos excipientes com o pico de resveratrol.

A linearidade de resposta do detector foi avaliada para as várias soluções padrões no intervalo de $0,4-10 \mu \mathrm{g} / \mathrm{mL}$. Os valores médios calculados para o coeficiente de correlação $\left(\mathrm{r}^{2}=0,9999, \mathrm{y}=\right.$ 173978.4x - 9990,8) indicaram linearidade da curva de calibração. A validade dos ensaios foi verificada por meio da análise da variância (ANOVA), a qual confirmou a linearidade do método (Fcal > Fcrítico; $p>0,05)$. Os valores de LD e LQ calculados foram 0,09 e $0,26 \mu \mathrm{g} /$ $\mathrm{mL}$, respectivamente, indicando a sensibilidade do método para a quantificação do resveratrol nos sistemas nanoestruturados lipídicos.

O ensaio de exatidão foi realizado por meio da avaliação da recuperação do resveratrol após contaminação das suspensões coloidais brancas com uma solução padrão do fármaco em três diferentes níveis. Os resultados mostraram a inexistência de diferenças significativas entre os valores de concentração de resveratrol teóricos e encontrados. Os valores de recuperação obtidos variaram entre 97,5-101,73\%, satisfazendo os critérios de aceitação do estudo. Os valores de DPR obtidos na avaliação da precisão do método foram menores que 5\%, indicando uma variabilidade intradia e interdia satisfatória. A quantidade de resveratrol encontrada em 3 dias diferentes foi equivalente para todas as suspensões coloidais testadas $(\mathrm{P}<0,05)$. O conjunto dos resultados de validação indicou que o método de cromatografia em fase reversa desenvolvido para determinação do resveratrol nos sistemas nanoestruturados lipídicos é especifico, linear, exato e preciso.

\section{Caracterização físico-química das suspensões coloidais}

A Tabela 1 demonstra as características de tamanho e potencial zeta das suspensões coloidais preparadas na ausência e na presença de diferentes quantidades iniciais de resveratrol. Como pode ser observado, o diâmetro médio das partículas das suspensões coloidais brancas variou em função do tipo de nanocarreador lipídico, sendo de 221, 119 e $22 \mathrm{~nm}$ para as $\mathrm{NLS}_{\mathrm{b}}, \mathrm{NE}_{\mathrm{b}}$ e $\mathrm{ME}_{\mathrm{b}}$, respectivamente. A adição do resveratrol às formulações não afetou o diâmetro médio das partículas. O índice de polidispersão, que fornece informações sobre a homogeneidade da distribuição dos tamanhos, foi baixo $(<0,3)$ para todas as dispersões obtidas, indicando a formação de sistemas monodispersos. As análises de potencial zeta indicaram a obtenção de partículas de carga negativa, decorrentes da presença da lecitina, sendo observada uma redução nos valores de carga superficial nas microemulsões, provavelmente devido à presença de maiores concentrações de Solutol HS 15, um surfactante não ionizável, na interface das partículas. Apesar das variações observadas nos valores do potencial zeta das formulações preparadas com fármaco, não foi possível afirmar a existência de algum efeito da quantidade inicial de fármaco sobre a carga superficial das partículas.

\section{Determinação do teor, eficiência de encapsulação e recuperação do resveratrol}

Após a validação do método analítico e análise das suspensões coloidais por CLAE, o teor, a eficiência de encapsulação e recuperação do resveratrol foram estimados. A eficiência de encapsulação foi maior que $94 \%$ para todas as formulações testadas, demonstrando que a maior parte do fármaco se encontra associado ao nanocarreador. Entretanto, os valores de teor de fármaco variaram de 186,9 a 852,8 $\mu \mathrm{g} / \mathrm{mL}$, dependendo da formulação testada (Tabela 2).
Tabela 1. Caracterização físico-química dos sistemas nanoestruturados lipídicos $(\mathrm{n}=3)$

\begin{tabular}{cccc}
\hline $\begin{array}{c}\text { Quantidade inicial } \\
\text { de resveratrol }\end{array}$ & $\begin{array}{c}\text { Tamanho }(\mathrm{nm}) \\
(\mathrm{IP})^{\mathrm{a}}\end{array}$ & $\begin{array}{c}\text { Potencial Zeta } \\
(\mathrm{mV})^{\mathrm{a}}\end{array}$ \\
\hline Sem fármaco & $\mathrm{NLS}_{\mathrm{b}}$ & $221,1 \pm 18,4(0,255 \pm 0,010)$ & $-35,3 \pm 4,1$ \\
& $\mathrm{NE}_{\mathrm{b}}$ & $119,2 \pm 2,5(0,115 \pm 0,016)$ & $-38,53 \pm 0,7$ \\
& $\mathrm{ME}_{\mathrm{b}}$ & $22,9 \pm 3,8(0,267 \pm 0,02)$ & $-15,8 \pm 0,6$ \\
$5 \mathrm{mg}$ & $\mathrm{NLS}_{\mathrm{r}}$ & $223,9 \pm 10,7(0,236 \pm 0,02)$ & $-32,7 \pm 3,2$ \\
& $\mathrm{NE}_{\mathrm{r}}$ & $113,8 \pm 5,8(0,121 \pm 0,03)$ & $-28,3 \pm 4,5$ \\
& $\mathrm{ME}_{\mathrm{r}}$ & $21,4 \pm 4,0(0,311 \pm 0,05)$ & $-12,1 \pm 1,6$ \\
$10 \mathrm{mg}$ & $\mathrm{NLS}_{\mathrm{r}}$ & $193,2 \pm 5,1(0,209 \pm 0,02)$ & $-33,2 \pm 2,8$ \\
& $\mathrm{NE}_{\mathrm{r}}$ & $112,3 \pm 0,8(0,122 \pm 0,07)$ & $-41,3 \pm 2,9$ \\
& $\mathrm{ME}_{\mathrm{r}}$ & $19,19 \pm 0,5(0,186 \pm 0,03)$ & $-18,1 \pm 0,8$ \\
$15 \mathrm{mg}$ & $\mathrm{NLS}_{\mathrm{r}}$ & $203,5 \pm 9,0(0,278 \pm 0,05)$ & $-29,3 \pm 3,0$ \\
& $\mathrm{NE}_{\mathrm{r}}$ & $113,1 \pm 5,0(0,124 \pm 0,02)$ & $-35,8 \pm 3,4$ \\
& $\mathrm{ME}_{\mathrm{r}}$ & $19,88 \pm 0,8(0,222 \pm 0,03)$ & $-17,0 \pm 2,7$ \\
\multirow{4}{*}{$20 \mathrm{mg}$} & $\mathrm{NLS}_{\mathrm{r}}$ & $228,0 \pm 10,8(0,254 \pm 0,02)$ & $-34,2 \pm 2,2$ \\
& $\mathrm{NE}_{\mathrm{r}}$ & $117,7 \pm 2,2(0,127 \pm 0,01)$ & $-40,6 \pm 2,7$ \\
& $\mathrm{ME}_{\mathrm{r}}$ & $19,37 \pm 0,3(0,178 \pm 0,03)$ & $-17,0 \pm 2,6$ \\
\hline
\end{tabular}

Entre parêntesis: índice de polidispersão (IP)

Tabela 2. Resultados de teor e recuperação do resveratrol nas suspensões coloidais de nanocarreadores lipídicos $(\mathrm{n}=3)$

\begin{tabular}{cccc}
\hline $\begin{array}{c}\text { Quantidade inicial de } \\
\text { resveratrol }\end{array}$ & $\begin{array}{c}\text { Teor de resveratrol } \\
(\mu \mathrm{g} / \mathrm{mL})^{*}\end{array}$ & $\begin{array}{c}\text { Recuperação } \\
(\%)\end{array}$ \\
\hline $5 \mathrm{mg}$ & $\mathrm{NLS}_{\mathrm{r}}$ & $186,9 \pm 14,9^{\mathrm{a}}$ & $74,76 \pm 5,95$ \\
& $\mathrm{NE}_{\mathrm{r}}$ & $190,1 \pm 12,4^{\mathrm{a}}$ & $79,04 \pm 0,85$ \\
& $\mathrm{ME}_{\mathrm{r}}$ & $199,0 \pm 8,0^{\mathrm{a}}$ & $79,93 \pm 3,20$ \\
\multirow{4}{*}{$10 \mathrm{mg}$} & $\mathrm{NLS}_{\mathrm{r}}$ & $350,7 \pm 19,4^{\mathrm{b}}$ & $70,15 \pm 3,87$ \\
& $\mathrm{NE}_{\mathrm{r}}$ & $415,2 \pm 20,3^{\mathrm{c}}$ & $83,05 \pm 4,06$ \\
& $\mathrm{ME}_{\mathrm{r}}$ & $412,2 \pm 13,5^{\mathrm{c}}$ & $82,45 \pm 2,69$ \\
& $\mathrm{NLS}_{\mathrm{r}}$ & $212,1 \pm 9,6^{\mathrm{a}}$ & $28,28 \pm 1,29$ \\
& $\mathrm{NE}_{\mathrm{r}}$ & $338,8 \pm 20,2^{\mathrm{d}}$ & $45,17 \pm 2,69$ \\
& $\mathrm{ME}_{\mathrm{r}}$ & $619,3 \pm 25,2^{\mathrm{e}}$ & $82,03 \pm 3,36$ \\
& $\mathrm{NLS}_{\mathrm{r}}$ & $206,3 \pm 35,4^{\mathrm{a}}$ & $20,63 \pm 3,54$ \\
& $\mathrm{NE}_{\mathrm{r}}$ & $327,0 \pm 6,0^{\mathrm{d}}$ & $32,70 \pm 0,60$ \\
& $\mathrm{ME}_{\mathrm{r}}$ & $852,8 \pm 3,46^{\mathrm{f}}$ & $85,28 \pm 0,35$ \\
\hline
\end{tabular}

*Letras diferentes indicam diferenças significativas entre as médias $(\mathrm{p}<0,001)$.

A análise estatística demonstrou que o teor de fármaco variou significativamente em função do tipo de nanocarreador (NLS, NE e ME) e da quantidade inicial de resveratrol, visto que valores de $\mathrm{F}_{\text {calculado }}$ foram maiores que $\mathrm{F}_{\text {tabelado }}(\alpha=0,05)$ (Tabela 3).

Após a aplicação do teste de Tukey, verificou-se que com a menor quantidade de fármaco ( $5 \mathrm{mg}$ ) não houve diferenças significativas entre os teores encontrados nas suspensões coloidais de NLS, NE e ME. Diferenças foram encontradas entre os carreadores quando a concentração inicial de fármaco foi igual ou maior que $10 \mathrm{mg}$, com valores de recuperação variando entre 70 e $80 \%$. Contudo, com a adição de 15 ou $20 \mathrm{mg}$ de resveratrol, os valores de recuperação nas NLS e NE reduziram drasticamente, enquanto se mantiveram elevados para as ME. Entre os três tipos de carreadores, as NLSs demonstraram uma menor capacidade de incorporar o resveratrol. De fato, as NLS têm demonstrado uma limitada capacidade de incorporar fármacos, 
Tabela 3. Resultados da análise da variança realizada para avaliar o efeito do nanocarreador e da quantidade inicial de resveratrol sobre o teor de fármaco nas suspensões coloidais

\begin{tabular}{cccccc}
\hline Origem da variação & $\begin{array}{c}\text { Soma dos } \\
\text { quadrados }\end{array}$ & $\begin{array}{c}\text { Graus de } \\
\text { liberdade }\end{array}$ & $\begin{array}{c}\text { Quadrado } \mathrm{F}_{\text {calculado }}{ }^{*} \\
\text { médio }\end{array}$ & $\mathrm{F}_{\text {crítico }}{ }^{*}$ \\
\hline $\begin{array}{c}\text { Tipo do nanocarreador } \\
\text { Quantidade inicial de } \\
\text { resveratrol }\end{array}$ & 304294,5 & 2 & 252147,2 & 785,2 & 3,40 \\
Interação & 467852,3 & 6 & 77975,3 & 242,8 & 2,50 \\
Dentro dos grupos & 7706,8 & 24 & 321,1 & & \\
Total & 1343776,4 & 35 & 38393,6 & & \\
\hline
\end{tabular}

$* \alpha=0,05$

devido à baixa solubilidade das substâncias na fase oleosa fundida e à formação de um cristal perfeito após a solidificação das partículas, o qual é constituído de arranjos de lipídio altamente organizados que limitam o espaço para a acomodação das moléculas ou cristais de fármaco. ${ }^{31,48,49}$ Por outro lado, para as microemulsões, os valores de recuperação elevados evidenciaram a alta capacidade de tais sistemas em solubilizar fármacos hidrofóbicos. De fato, as microemulsões podem incorporar grandes quantidades de fármacos devido à alta capacidade de solubilização. ${ }^{50}$ De acordo com os resultados, o teor máximo de resveratrol encontrado nas suspensões coloidais foi de cerca de 350,415 e $852 \mu \mathrm{g} / \mathrm{mL}$ para as NLS, NE e ME, correspondendo a 7; 8,3 e $17 \%$ de fármaco em relação à massa de lipídio empregada nas formulações, respectivamente.

Considerando a solubilidade aquosa do resveratrol que é de unicamente $0,03 \mathrm{mg} / \mathrm{mL}$, a obtenção das microemulsões permitiu o aumento da concentração deste fármaco em até 28,4 vezes, podendo esta concentração ainda ser maior, visto que a adição de quantidades iniciais maiores de resveratrol não foram testadas.

O teor de resveratrol também foi investigado após a incorporação da HEC nas suspensões coloidais. Os valores encontrados foram de $359,2 \pm 23,8 ; 430,1 \pm 18,4$ e 425,7 $\pm 20,2$ para $\mathrm{NLS}_{\mathrm{rv}} \mathrm{NE}_{\mathrm{rv}}$, e $\mathrm{ME}_{\mathrm{rv}}$, respectivamente, quando $10 \mathrm{mg}$ de resveratrol foram adicionados às formulações. Para as microemulsões $\left(\mathrm{ME}_{\mathrm{rv}}\right)$ preparadas com uma quantidade inicial de 15 e $20 \mathrm{mg}$, os valores foram de $637,8 \pm 22,9$ e $859,2 \pm 6,1$, respectivamente. Estes valores foram similares aos encontrados nas suspensões coloidais, conforme demonstrado na Tabela 2.

\section{Avaliação da morfologia dos hidrogeis}

As fotomicrografias obtidas a partir dos filmes formados a partir dos hidrogeis encontram-se demonstradas na Figura 2. A presença de partículas dispersas na matriz polimérica da HEC pode ser observada em todas as amostras analisadas. Entretanto, diferenças na morfologia das partículas podem ser verificadas nas micrografias. Enquanto os filmes contendo a nanoemulsão e a microemulsão exibem estruturas de formato esférico, o filme contendo nanopartículas lipídicas sóli- das é caracterizado por apresentar partículas com faces quadradas, característico de sistemas isométricos cúbicos. Isto provavelmente decorre do fato da nano e microemulsão serem formadas a partir de um lipídio líquido (óleo de rícino), enquanto que a nanopartícula lipídica sólida é formada a partir de um lipídio sólido à temperatura ambiente, em que as moléculas do mesmo são dispostas de maneira mais organizada na estrutura da partícula.

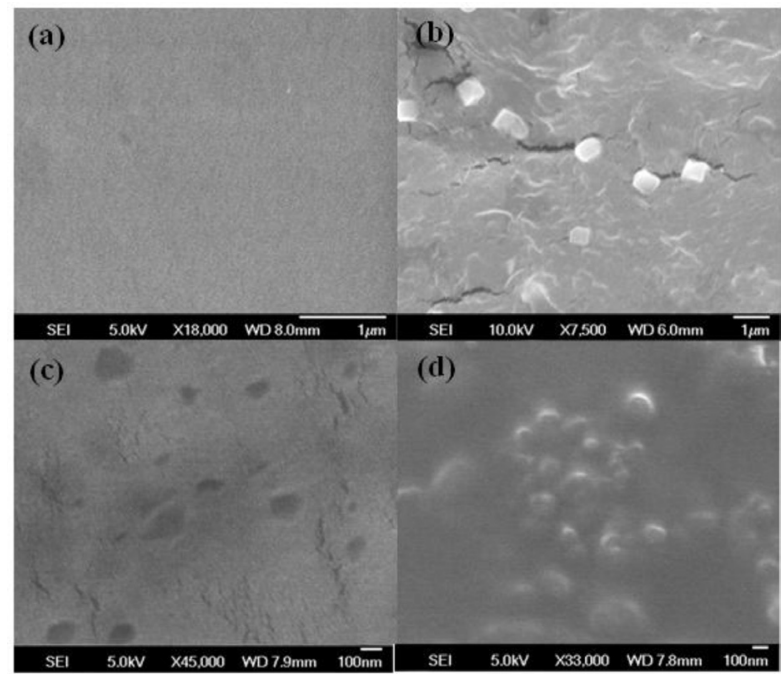

Figura 2. Fotomicrografias dos filmes obtidos a partir dos hidrogeis de HEC obtidas por microscopia eletrônica de varredura com emissão de campo (MEVFEG). (a) Gel branco; (b) hidrogel-NLS; (c) hidrogel-NE; (d) hidrogel-ME

\section{Análise reológica dos hidrogeis de HEC}

Os geis contendo os diferentes nanocarreadores lipídicos e o gel puro (sem nanocarreadores) exibiram comportamento não newtoniano, em que a viscosidade dependeu da tensão de cisalhamento aplicada (Tabela 4). Nestes resultados foi verificada a redução da viscosidade dos hidrogeis com o aumento da tensão de cisalhamento, característico de fluidos que apresentam comportamento pseudoplástico. Por outro lado, foi observado um aumento da viscosidade dos geis contendo os nanocarreadores lipídicos em relação ao gel puro, sendo que o aumento mais pronunciado ocorreu nos geis contendo NLS, provavelmente devido à presença de partículas sólidas na preparação. Entretanto, estudos mais aprofundados são ainda necessários para compreender as interações existentes entre agente gelificante e nanocarreador.

\section{CONCLUSÃO}

NLSs, NEs e MEs contendo resveratrol foram obtidas por um método simples de formação de emulsão e difusão do solvente a quente, variando somente a composição da formulação. O método

Tabela 4. Resultados obtidos na caracterização do gel de HEC puro e contendo os nanocarreadores lipídicos

\begin{tabular}{|c|c|c|c|c|c|c|c|c|c|}
\hline \multirow{2}{*}{$\begin{array}{l}\text { Velocidade de } \\
\text { rotação (rpm) }\end{array}$} & \multirow{2}{*}{$\begin{array}{c}\text { Gradiente de } \\
\text { cisalhamento } \\
\qquad\left(\mathrm{s}^{-1}\right)\end{array}$} & \multicolumn{2}{|c|}{ Gel de HEC puro } & \multicolumn{2}{|c|}{ hidrogel-NLS } & \multicolumn{2}{|c|}{ hidrogel-NE } & \multicolumn{2}{|c|}{ hidrogel-ME } \\
\hline & & $\begin{array}{c}\tau \\
\left(\mathrm{D} / \mathrm{cm}^{2}\right)\end{array}$ & $\begin{array}{c}\eta \\
(\mathrm{cP})\end{array}$ & $\begin{array}{c}\tau \\
\left(\mathrm{D} / \mathrm{cm}^{2}\right)\end{array}$ & $\begin{array}{c}\eta \\
(\mathrm{cP})\end{array}$ & $\begin{array}{c}\tau \\
\left(\mathrm{D} / \mathrm{cm}^{2}\right)\end{array}$ & $\begin{array}{c}\eta \\
(\mathrm{cP})\end{array}$ & $\begin{array}{c}\tau \\
\left(\mathrm{D} / \mathrm{cm}^{2}\right)\end{array}$ & $\begin{array}{c}\eta \\
(\mathrm{cP})\end{array}$ \\
\hline 5 & 6,6 & 6,2 & 94,2 & 9,3 & 141,6 & 6,9 & 104,4 & 7,21 & 109,2 \\
\hline 10 & 13,2 & 12,6 & 95,4 & 17,6 & 133,8 & 13,5 & 102,6 & 13,8 & 104,4 \\
\hline 15 & 26,2 & 25,0 & 94,8 & 33,7 & 127,8 & 26,5 & 100,3 & 26,9 & 102,0 \\
\hline 20 & 39,6 & 38,8 & 93,0 & nd & nd & 39,9 & 98,4 & 39,2 & 99,8 \\
\hline
\end{tabular}

Nd: Não determinado; Torque $>100 \%$. $\tau$ : Tensão de cisalhamento, $\eta$ : viscosidade 
de CLAE desenvolvido para quantificar o resveratrol nas suspensões e hidrogeis demonstrou ser específico, linear, exato e preciso. Apesar de terem sido obtidos valores de eficiência de encapsulação elevados em todas as formulações, o teor de resveratrol variou significativamente em função do tipo de carreador e da quantidade inicial de fármaco adicionado na fase orgânica da preparação. Dentre os três tipos de nanocarreadores lipídicos, as MEs exibiram uma maior capacidade de incorporar o resveratrol, sendo que a concentração final na dispersão foi cerca de 28 vezes maior que a solubilidade aquosa do mesmo. A incorporação de HEC na fase aquosa das suspensões coloidais proporcionou a obtenção de sistemas pseudoplásticos. A presença dos nanocarreadores conduziu ao aumento da viscosidade do gel de HEC, sobretudo quando as NLSs foram empregadas. Os resultados indicaram que as suspensões coloidais lipídicas obtidas neste estudo podem potencialmente ser exploradas como carreadores para administração tópica do resvetrarol.

\section{REFERÊNCIAS}

1. Ignatowicz, E.; Baer-Dubowska W.; Pol. J. Pharmaco. 2001, 53, 557.

2. Burns, J.; Yokota, T.; Ashihara, H.; Lean, M. E. J.; Crozier, A.; J. Agric. Food Chem. 2002, 50, 3337.

3. Fulda, S.; Debatin, K. M.; Cancer Detect Prev. 2006, 30, 217.

4. Yu, C.; Shin, Y. G.; Chow, A.; Li, Y.; Kosmeder, J. W.; Lee, W. S.; Hirschelman, W. H.; Pezzuto, J. M.; Mehta, R. G.; van Breemen, R. B.; Pharm Res. 2002, 19, 1907.

5. Vitrac, X.; Desmoulière, A.; Brouillaudc, B.; Krisa, S.; Deffieux, G.; Barthe, N.; Rosenbaumb, J.; Mérillon, J. M.; Life Sci. 2003, 72, 2219.

6. Shankar, S.; Singh, G.; Srivastava, R. K.; Front Biosci. 2007, 12, 4839.

7. Ulrich, S.; Wolter, F.; Stein, J. M.; Mol. Nutr. Food Res. 2005, 49, 452.

8. Bove, K.; Lincoln, D. W.; Tsan, M. F.; Biochem. Biophys. Res. Commun 2002, 291, 1001.

9. Asensi, M.; Medina, I; Ortega, A.; Carretero, J.; Baño, M. C.; Obrador, E.; Estrela, J. M.; Free Radical Biol. Med. 2002, 33, 387.

10. Gautam, S. C.; Xu, Y. X.; Dumaguin, M.; Janakiraman, N.; Chapman, R. A.; Bone Marrow Transplant 2000, 25, 639.

11. Fuhrman, B.; Lavy, A.; Aviram, M.; Am. J. Clin. Nutr. 1995, 61, 549.

12. Rastija, V.; Medic-Saric, M.; Eur. J. Med. Chem. 2009, 44, 400.

13. Olas, B.; Wachowicz, B.; Platelets 2005, 16, 251.

14. Jung, H. J.; Seu, Y. B.; Lee, D. G.; J. Microbiol. Biotechnol. 2007, 17, 1324.

15. Martín, A. R.; Villegas, I.; Sánchez-Hidalgo, M.; Lastra, C. A.; Br. J. Pharmacol. 2006, 147, 873.

16. Gusman, J.; Malonne, H.; Atassi, G.; Carcinogenesis 2001, 22, 1111.

17. Athar, M.; Back, J. H.; Tang, X.; Kim, K. H.; Kopelovich, L.; Bickers, D. R.; Kim, A. L.; Toxicol. Appl. Pharmacol. 2007, 224, 274.

18. Stivala, L. A.; Savio, M.; Carafoli, F.; Perucca, P.; Bianchi, L.; Maga, G.; Forti, L.; Pagnoni, U. M.; Albinii, A.; Prosperi, E.; Vannini, V.; J. Biol. Chem. 2001, 276, 22586.

19. Williamson, G.; Manach, C.; Am. J. Clin. Nutr. 2005, 81, 243S.

20. Walle, T.; Hsieh, F.; Delegge, M. H.; Oatis Jr., J. E.; Walle, U. K.; Drug Metab. Dispos. 2004, 32, 1377.

21. Wenzel, E.; Somoza, V.; Mol. Nutr. Food Res. 2005, 49, 472.

22. Piñeiro, Z.; Palma, M.; Barroso, C. G.; J. Chromatogr., A 2006, 1110 , 61.
23. Janga, M.; Pezzuto, J. M.; Cancer Lett. 1998, 134, 81.

24. Soleas, G. J.; Grassc, L.; Josephyd, P. D.; Goldbergb, D. M.; Diamandis, E. P.; Clin. Biochem. 2002, 35, 119.

25. Aziz, M. H.; Afaq, F.; Ahmad, N.; Photochem. Photobiol. 2005, 81, 25.

26. Afaq, F.; Adhami, V. M.; Ahmad, N.; Toxicol. Appl. Pharmacol. 2003, $186,28$.

27. Chan, M. M.; Biochem Pharmacol. 2002, 63, 99.

28. Docherty, J. J.; Smith, J. S.; Fu, M. M.; Stoner, T. S.; Booth, T.; Antiviral Res. 2004, 61, 19.

29. Hung, C. F.; Lin, Y. K.; Huang, Z. R.; Fang, J. Y.; Biol. Pharm. Bull. 2008, 31, 955 .

30. Wang, J. X.; Sun, X.; Zhang, Z. R.; Eur. J. Med. Chem. 2002, 54, 285.

31. Wissing, S. A.; Kayser, O.; Müller, R. H.; Adv. Drug Deliv. Rev. 2004, $56,1257$.

32. Manjunath, K.; Reddy, S. J.; Venkateswarlu, V.; Methods Find Exp. Clin. Pharmacol. 2005, 27, 127.

33. Vicentini, F. T. M. C.; Simi, T. R. M.; Del Ciampo, J. O.; Wolga, N. O.; Pitol, D. L.; Iyomasa, M. M.; Bentley, M. V. L. B.; Fonseca, M. J. V.; Eur. J. Med. Chem. 2008, 69, 948.

34. Shishu, S.; Rajan, K.; AAPS Pharm. Sci. Tech. 2009, 10, 559.

35. Careri, M.; Corradini, C.; Elviri, L.; Nicoletti, I.; Zagnoni, I.; J. Agric. Food Chem. 2003, 51, 5226.

36. Quirós, A. R. B.; López-Hernández, J.; Ferraces-Casais, P.; Lage-Yusty, M. A.; J. Sep. Sci. 2007, 30, 1262.

37. Wang, D.; Xu, Y.; Liu, W.; Phytomedicine 2008, 15, 859.

38. He, H.; Chen, X.; Wang, G.; Wang, J.; Davey, A. K.; J. Chromatogr., B: Anal Technol. Biomed. Life Sci. 2006, 832, 177.

39. Hu, F.; Jiang, S.; Du, W.; Yuan, H.; Ye, Y.; Zeng, S.; Int. J. Pharm. 2006, $314,83$.

40. Tandon, V.; Bhagavatula, S. K.; Nelson, W. C.; Kirby, B. J.; Electrophoresis 2008, 29, 1092.

41. http://www.ich.org/cache/compo/276-254-1.html, acessada em Setembro 2010.

42. U.S. Pharmacopeia, $23^{\text {th }}$ ed., U.S. Pharmacopeial Convention: Rockville, 1995.

43. Purdon, C. H.; Azzi, C. G.; Zhang, J.; Smith, E. W.; Maibach, H. I.; Crit. Rev. Ther. Drug. Carrier Syst. 2004, 21, 97.

44. Rizwan, M.; Aqil, M.; Talegaonkar, S.; Azeem, A.; Sultana, Y.; Ali, A.; Recent Pat. Drug. Deliv. Formul. 2009, 59, 427.

45. Bouwstra, J. A.; Honeywell-Nguyen, P. L.; Adv. Drug Deliv. Rev. 2002, $54, \mathrm{~S} 41$.

46. Schäfer-Korting, M.; Mehnert, W.; Korting, H. C.; Adv. Drug Deliv. Rev. 2007, 59, 427.

47. Dora, C. L.; Silva, L. F. C.; Soldi, V.; Pignot-Paintrand, I.; Borsali, R.; Lemos-Senna, E.; Abstracts of the $36^{\text {th }}$ Annual Meeting and Exposition of the Controlled Release Society, Copenhagen, Denmark, 2009.

48. Westesen, K.; Bunjes, H.; Koch, M. H. J.; J. Controlled Release 1997, $48,223$.

49. Garcia-Fuentes, M.; Alonso, M. J.; Torres, D.; J. Colloid Interface Sci. 2005, 285, 590 .

50. Nazar, M. Z.; Khan, A. M.; Shah, S. S.; AAPS Pharm. Sci. Tech. 2009, $10,1286$. 